\title{
La Neurotecnología Educativa. Claves del uso de la tecnología en el proceso de aprendizaje
}

\author{
Silvia Pradas Montilla. Universidad Internacional de la Rioja \\ Recepción: 10 de Junio de 2017 | Aceptado: 16 de Junio de 2017 \\ Correspondencia: Silvia Pradas | Correo-e: silvia.pradas@unir.net \\ iD $0000-0002-8312-1080$ \\ Citar: Pradas, S. (2017). La Neurotecnología Educativa. Claves del uso de la tecnología en el \\ proceso de aprendizaje. ReiDoCrea, 6(2), 40-47.
}

\begin{abstract}
Resumen: La neurotecnología educativa es el enfoque del uso de la tecnología en el ámbito educativo interpretando adecuadamente el procesamiento neuronal. En definitiva, una nueva ciencia del aprendizaje, con base en el conocimiento sobre el funcionamiento del cerebro humano y la metodología utilizada en el empleo de la tecnología en el aula. Una metodología centrada más en el "cómo" del aprendizaje, que en "qué". En un mundo caracterizado por la diversidad de fuentes de acceso a los contenidos, noticias y datos y sobreabundancia de información, requiere una concepción del aprendizaje enfocado hacia procesos de indagación, de coordinación y articulación dinámica del conocimiento para la resolución de problemas más que adquisición de conocimientos fijos, cerrados y estancos. Partimos del conocimiento del cerebro y de qué manera entra la información al cerebro y cómo éste la procesa. De esa manera comprendemos los mecanismos de la actividad mental y la conducta humana. En consecuencia, ese conocimiento se convierte en algo inestimable para ayudar a nuestros alumnos en su proceso de aprendizaje y diseñar nuevos modelos metodológicos que devuelvan el protagonismo del proceso al alumno. Desde esta perspectiva, la neurotecnología educativa diseñará estrategias, catalogará software y aplicará metodologías que den respuestas al desarrollo cognitivo y la maduración cerebral, a los diferentes aprendizajes y a las dificultades y trastornos que observamos en los estudiantes en edad escolar. Así como el desarrollo del pensamiento computacional, de las funciones ejecutivas o de las inteligencias múltiples a través de la tecnología. Finalmente, abordaremos las soluciones que nos ofrece la tecnología para la resolución y mejora en el proceso del aprendizaje.
\end{abstract}

Palabras clave: Neuropsicología | Tecnología

\section{Educational Neurotechnology. Keys to the use of technology in the learning process}

\begin{abstract}
Educational neurotechnology is the approach to uses of technology in the educational area and involves a correct understanding of the neuronal processing. It is definitely a new educational science with its basis in the knowledge about the human brain functioning and a methodology regarding the use of technology in the teaching space. The methodology is centred in the "how" the learning is achieved more than in "what" it is learned. A word characterized by the diversity of sources in the access to contents, news, data, and overabundance of information, requires a concept of learning focused into inquire, coordination and dynamic articulation of knowledge for the problem solving more than the acquisition of fixed, closed knowledge. We depart from the comprehension of the brain, and how the information is processed. Thus, we can understand the mechanism of human mental activity and behaviour. In consequence, the knowledge is converted into something inestimable to help our students in their process of learning and to design new methodological models that will bring back prominence of the process to students. From this point of view, educational neurotechnology will design strategies, will catalogue software and will apply methodologies that will give answers to the cognitive development and brain maturation to the different learning and difficulties or disorders we can observe among students. In addition, computational thinking, executive functions or multiple intelligences will be developed throughout technology. Finally, we will account for the solutions offered by technology for the resolution and improvement of the learning processes.
\end{abstract}

Keywords: Neuropsychology | Technology 


\section{Introducción}

Hay quien ya habla de una nueva era, la era de la neurotecnología, en la que las nuevas ciencias cognitivas y los nuevos desarrollos en neurociencia aportan su conocimiento para diseñar nuevas tecnologías informáticas, inteligentes y robóticas.

Si al término de neurotecnología le acompaña el término educativa lo que se quiere transmitir es la idea de los nuevos enfoques que permitan abordar el aprendizaje desde una orientación científico multidisciplinar. La comprensión del cerebro y su estudio profundo resalta la imperiosa necesidad de vincular las ciencias del aprendizaje y la investigación cerebral, en un afán por lograr una visión interdisciplinaria que permita conjuntar los avances y la tecnología en la comprensión del aprendizaje humano.

La neurotecnología educativa es el enfoque del uso de la tecnología en el ámbito educativo interpretando adecuadamente el procesamiento neuronal. En definitiva, una nueva ciencia del aprendizaje, con base en el conocimiento sobre el funcionamiento del cerebro humano y la metodología utilizada en el empleo de la tecnología en el aula. Una metodología centrada más en el "cómo" del aprendizaje que en el "qué". En un mundo caracterizado por la diversidad de fuentes de acceso a los contenidos, las noticias y los datos y la sobreabundancia de información, se requiere una concepción del aprendizaje enfocado hacia procesos de indagación, de coordinación y articulación dinámica del conocimiento para la resolución de problemas más que de adquisición de conocimientos fijos, cerrados y estancos.

La clave estará en conocer las ventajas o beneficios de usar la tecnología respecto a nuestro cerebro, como el descubrir sus peligros para desarrollar nuevas estrategias. Por ejemplo, saber gracias al neurólogo e investigador Gary Small que el uso de Internet tiene resultados positivos para el funcionamiento del cerebro, aunque el problema surge cuando se exagera su uso. Los sujetos que pasan alrededor de diez horas al día frente al ordenador pueden ver reducidas sus aptitudes para el contacto personal, como mantener una conversación cara a cara.

Partimos de la idea de que todos "los procesos cognitivos siempre se realizan en el cerebro", como afirmaron Stern, Grabner, Schumacher, Neuper y Saalbach (2005, p.12) después de efectuar un extenso estudio sobre neurociencias pedagógicas. Agregan que "es indispensable un diálogo entre las neurociencias y la investigación educacional". Las investigaciones hasta ahora nos demuestran que el aprendizaje escolar produce un aumento de la eficiencia neural, porque activa selectivamente algunas regiones del cerebro, lo que permite asimilar con mayor eficiencia diferentes informaciones y conocimientos. En consecuencia, nosotros como profesionales de la educación debemos conocer la estimulación de las áreas cerebrales involucradas en procesos como la lectura, la escritura o las matemáticas, y especialmente en el lenguaje, en la percepción visual y fonológica, en la orientación espacial y secuencial, en la memoria verbal y en la coordinación grafomotriz, para lograr aprendizajes más efectivos.

Coincidimos con Dehaene (2007, p.22) en que los progresos de las neurociencias y de la psicología cognitiva han originado el nacimiento de una "verdadera neurociencia de la educación en la frontera entre la psicología y la medicina, capacitada para aprovechar las nuevas imágenes del cerebro con la finalidad de optimizar las estrategias de enseñanza y adaptarlas a cada cerebro de niño o de adulto". MacCandliss (2010) también considera que las neurociencias educacionales requieren investigar los cambios que se producen en el cerebro con el aprendizaje. 
Entendemos que conocer cómo se construye y madura el cerebro se convierte en algo fundamental para entender cómo funciona, y es el camino más adecuado para comprender los mecanismos de la actividad mental y la conducta humana. En consecuencia, es una ayuda inestimable para ayudar a nuestros alumnos en su proceso de aprendizaje.

Si queremos diseñar una hoja de ruta para la ayuda de nuestros alumnos en su proceso de aprendizaje desde la perspectiva de la neuropsicología y de la tecnología, abordaremos el tema desde tres enfoques. En primer lugar, el cambio metodológico que implica, es decir, el conocimiento del funcionamiento del cerebro nos invita a aplicar estrategias didácticas acordes. En segundo lugar, aplicación de la tecnología en el proceso siempre con una intención pedagógica y en el momento oportuno con los recursos adecuados que respondan a esa intención. Y en tercer lugar, servirnos de los avances tecnológicos para personalizar el aprendizaje.

\section{E L C A M B IO ME T O D O L Ó G I C O}

Entendemos que un educador que tiene conocimientos acerca del cerebro, que conoce algunas de las particularidades del sistema nervioso y del cerebro, entenderá cómo el ser humano se desarrolla y cómo el aprendizaje influye en este proceso. Reyes (2016) afirma que, al comprender cómo aprende el cerebro, automáticamente repensaremos el papel que desempeñamos como agentes educativos y buscaremos mejorar la calidad de nuestro trabajo y de nuestros programas, la metodología que usamos y la calidad de las actividades que proponemos. Este conocimiento nos llevará a actuar con mayor efectividad y creatividad, en pro de lo que realmente debemos hacer: propiciar el desarrollo del individuo y de su potencial humano.

La cultura y la sociedad actual demandan un alumno creativo, crítico, capaz de pensar, razonar y abstraer, que resuelve problemas, que es capaz de diseñar, desarrollar y evaluar proyectos colaborativamente, que aprenda a construir las estructuras mentales que condicionen su aprender a aprender (Sánchez, 2000). En la educación de la Era Virtual, el énfasis debe estar en cómo aprender más que en qué aprender. Los maestros no solo deben preocuparse de cómo enseñar, sino de cómo los alumnos aprenden. Un mundo caracterizado por la diversidad de fuentes de acceso a los contenidos, noticias y datos y la sobreabundancia de información, requiere una concepción del aprendizaje como un proceso de indagación, de coordinación y de articulación dinámica del conocimiento para la resolución de problemas más que de adquisición de conocimientos fijos, cerrados y estancos.

La Educación tiene que elaborar un nuevo modelo que se centre en determinar cómo aprende los cerebros de los niños y cuáles serían las estrategias psicopedagógicas y neuropsicológicas más adecuadas para este aprendizaje.

La integración de la tecnología en el proceso de aprendizaje implica un cambio metodológico, poniendo de relieve la intencionalidad pedagógica del modelo, que implique, además, otro tipo de intencionalidad, la intencionalidad tecnológica.

El nuevo modelo incluirá elementos claves como:

$>\quad$ Contextualización. Definir el escenario o contexto en el que van a trabajar los alumnos y los profesores. La contextualización ofrece la primera señal, la de sentirse bien orientados. Esta fase es importante por varias razones. Una razón es porque el cerebro, por naturaleza, está diseñado para procesar y retener información con sentido. Otra es la motivación, del latín motivus (relativo al movimiento), que es aquello 
que mueve o tiene eficacia, es el motor de la conducta humana. En la contextualización se despierta el interés por una actividad, por una necesidad, que incita a la persona a la acción. Según Gilbert (2005): "El cerebro está diseñado para la supervivencia... Cuando nos enfrentamos a una situación de aprendizaje, hay una parte de nuestro cerebro que se pregunta: ¿Necesito este aprendizaje para sobrevivir? ¿Sí o no? En caso afirmativo podemos seguir con el aprendizaje. Sin embargo, si la respuesta es negativa, olvidémonos de todo". En este sentido, la motivación para el nuevo aprendizaje vuelve a considerarse como un factor determinante del mismo.

$>\quad$ Objetivos. Antes de aprender y, por tanto, antes de emplear la tecnología, el alumno debe tener claros los objetivos que trata de conseguir. Los objetivos son previsiones cognitivas de los resultados que se espera conseguir a lo largo del aprendizaje. El objetivo inmediato de todo aprendizaje es convertir la información en conocimiento para alcanzar la sabiduría. El conocimiento no se produce de una vez y para siempre, sino que es una elaboración, prolongada en el tiempo, de los conocimientos que permite descubrir en ellos nuevas perspectivas, implicaciones y, sobre todo, posibilidades de aplicación dentro y fuera del área correspondiente del saber. Los objetivos están estrechamente relacionados con los procesos de planeación, estrategias de trabajo, seriación y secuenciación, que junto con la memoria de trabajo, fluidez (diseño y verbal), solución de problemas complejos, flexibilidad mental, generación de hipótesis, constituyen los procesos que en su mayoría se consideran las funciones ejecutivas localizadas en la porción dorsal del cerebro (Stuss y Alexander, 2000). Por último, otro de los objetivos que tendremos en cuenta será el desarrollo de los valores que dignifiquen a nuestros alumnos a la hora de emplear la tecnología.

Profesor. El papel del profesor es igualmente trascendental e incluso determinante. A él le corresponde sensibilizarse hacia la nueva manera de enseñar en la que su papel no es ya el de transmitir conocimientos, sino el mucho más satisfactorio y gratificante de ayudar a los estudiantes a aprender. Se convierte en un facilitador o mediador del aprendizaje. El papel del profesor se diversifica a lo largo del aprendizaje. Por eso se puede hablar de las tareas del profesor antes, durante y después del proceso. En el antes, el papel del profesor es planificar las tareas, saber el estado de partida (dónde se encuentran actualmente) y el estado de meta (a dónde quiere que lleguen). En una palabra, visualiza la clase. En el durante, el profesor debe presentar los contenidos, plantear el trabajo, promover la comprensión, la retención y la transformación de los conocimientos. El profesor debe estimular, además, a los alumnos a ir más allá de lo adquirido, criticando y ponderando los conocimientos conseguidos o generando otros nuevos. Y en el después, el papel del profesor se centra, sobre todo, en la ayuda prestada a los alumnos para recuperar, transferir y evaluar los resultados del aprendizaje.

$>\quad$ Alumno. Al cambiar el modelo de aprendizaje hay que cambiar lógicamente el papel del alumno, es decir, su contribución a la tarea de aprender. Desde la perspectiva de la neuropsicología debemos añadir, en primer lugar, que cada cerebro humano es distinto a los otros y esto debe ser tomado en cuenta por quienes enseñamos. En segundo lugar, que cuando el cerebro aprende se modifica de manera plástica y que funciona de manera distinta a cada edad. Esas diferencias provienen de su genética, de sus procesos de socialización y del aprendizaje. El alumno no puede mantenerse pasivo en la escuela. Su participación activa es fundamental. El ámbito de esa participación es muy amplio y comienza con la propia disposición favorable, positiva hacia el aprendizaje, sigue con la planificación de las tareas correspondientes, el desarrollo de las estrategias adecuadas, la aplicación de los conocimientos adquiridos, y la evaluación de los resultados para desembocar en nueva propuesta de aprendizaje. 
$>\quad$ Instrumentos. La tecnología se convierte en instrumentos cognitivos que favorecen, desarrollan y mejoran las condiciones de la mente humana en la tarea de la construcción del conocimiento. En la actualidad contamos con hardware que permite emitir información visual, auditiva y táctil simultáneamente. Lo más importante de todos estos instrumentos no es que permitan adquirir información, sino que potencian, amplían y mejoran la capacidad humana para construir y generar conocimientos. Por eso, es interesante que el profesor señale qué tipo de instrumentos se pueden utilizar para realizar las actividades programadas y conseguir los objetivos propuestos en función del desarrollo neuropsicológico que busca. En este sentido, la catalogación del software que diseñe irá en la línea del desarrollo de los sistemas funcionales cerebrales.

$>\quad$ Procesos y actividades. El aprendizaje, especialmente el aprendizaje en un contexto tecnológico, implica necesariamente la puesta en marcha de una serie de procesos que llevan lógicamente a la construcción del conocimiento, al aprender a aprender. Estos procesos son: planificar la tarea, seleccionar y organizar la información, actuar de manera crítica y creativa, transferir y aplicar los conocimientos, etc. Este es el parámetro más importante de todo el arco del aprendizaje, ya que la calidad de los procesos iniciados por el estudiante determinará la calidad del aprendizaje realizado. Lo que más interesa a la hora de aprender con las TIC no es tanto el carácter exótico de las actividades que se puedan programar, sino el que puedan convertirse en verdaderas experiencias de aprendizaje para los alumnos. En todo caso, esas tareas no se pueden limitar a adquirir información. Serían tan aburridas como las tradicionales y desmotivarían a los alumnos a las pocas horas. Las tareas con tecnología deben permitir a los alumnos adquirir conocimientos, pero también, y sobre todo, desarrollar habilidades de comprensión y expresión, resolver problemas reales y auténticos, utilizar el pensamiento crítico o la creatividad, es decir, explorar, indagar, descubrir, comprobar o discutir. Eso sí puede conducir al alumno a conseguir el objetivo máximo, el desarrollo de las funciones ejecutivas y las habilidades del pensamiento con un equilibrio emocional adecuado.

$>\quad$ Evaluación. Por último, hay que pensar en la evaluación y los instrumentos de medida para evaluar el grado de consecución de los objetivos propuestos. La evaluación en este modelo de aprendizaje cobra toda su dimensión devolviendo a este proceso la trascendencia que no tiene en el aprendizaje tradicional. En este modelo no tiene sentido hacer una evaluación cuantitativa y centrada en los hechos, datos o conocimientos. Debe ser, por el contrario, una evaluación para aprender, hecha desde contextos múltiples, acercándose progresivamente a la evaluación tipificada como portfolio, en la que se evalúa especialmente la comprensión, la adquisición de estrategias, el aprender a aprender, la capacidad de autorregulación y las capacidades críticas o imaginativas.

En resumen, estos procesos de aprendizaje siguen la lógica del pensamiento que primero se prepara, luego planifica, analiza, critica, construye y evalúa. En este sentido, se entiende que aprender es pensar y que el aprendizaje no es más que el resultado del pensamiento que se enfrenta a una situación, proyecto o problema siguiendo las fases de su desarrollo. 

L A
TE C NOLO G Í A
E N
E L
P R O C E S O
D E

A P R E N D I Z A J E

Martín-Lobo (2016) afirma que los conocimientos de neuropsicología pueden llevar a diseñar y aplicar programas siguiendo el modelo neuropsicológico de Luria y la correspondencia del desarrollo cognitivo y la maduración cerebral, con los diferentes aprendizajes y con las dificultades y trastornos que observamos en los estudiantes en edad escolar:

- $\quad$ Programas de desarrollo de la atención.

- Programas para desarrollar las habilidades visuales, auditivas, táctiles y de integración sensorial para asegurar la calidad de la entrada de la información en el cerebro.

- $\quad$ Programas de motricidad, equilibrio y vestibulares, de desarrollo lateral y de sentido espacio-temporal para favorecer los procesos cerebrales relacionados con el aprendizaje.

- $\quad$ Programas de lenguaje, memoria y de habilidades superiores de pensamiento, de inteligencias múltiples, creatividad y Funciones Ejecutivas.

- Programas para superar las dificultades del aprendizaje, la dislexia, la discalculia, las dificultades del lenguaje.

- Programas para el autismo y otros.

La neurotecnología educativa diseñará estrategias, catalogará software y aplicará metodologías que den respuestas al desarrollo cognitivo y la maduración cerebral, con los diferentes aprendizajes, y a las dificultades y trastornos que observamos en los estudiantes en edad escolar.

Jiménez, Hernández, García y otros (2015) afirman que la atención selectiva y la atención sostenida están reguladas por estructuras corticales y subcorticales. Entre las estructuras corticales está la corteza prefrontal y las cortezas sensoriales. En el caso de las estructuras subcorticales, el tálamo óptico, el cuerpo estriado (núcleo caudado y lenticular), los núcleos septales y de Meynert y el cerebelo. En el caso del tálamo, se ha demostrado que existen neuronas que sólo responden a los estímulos novedosos y que se habitúan rápidamente (Jasper y Bertrand, 1966). A esto habría que añadir la corteza prefrontal, que juega un papel fundamental en el control voluntario de la atención.

El uso de la tecnología nos puede ayudar para mejorar la atención gracias al apoyo visual y auditivo que ofrece, porque prolongará la concentración durante más tiempo. La importancia de la atención en el proceso del aprendizaje es indiscutible, pero como cualquier otra habilidad, hay que desarrollarla a lo largo de la vida. De ahí que la clave estará en una buena selección de programas que ayuden a ejercitar la habilidad como, por ejemplo, actividades de buscar las diferencias. Seleccionar programas que trabajen: Atención visual, Atención auditiva, Tamaño, posición y color.

Bavelier y Davidson (2013) explican: "Cuando la gente juega con videojuegos de acción están reconfigurando la vía cerebral responsable del proceso visual. Estos videojuegos empujan al sistema visual humano hasta sus límites, y el cerebro se adapta a él. Y lo que el jugador aprende, lo traslada a otras actividades y posiblemente a la vida cotidiana".

Cuando el proceso de la integración sensorial se presenta desordenado se hacen evidentes una serie de problemas de aprendizaje, desarrollo y comportamiento. 
Gracias a la tecnología actual con la que contamos en el aula podemos exponer, siempre con una intención educativa, a los alumnos a una tormenta de estímulos, visuales-auditivos y táctiles, que alteren beneficiosamente sus circuitos cerebrales. Una situación de aprendizaje que favorece lo anteriormente dicho es el trabajo de los alumnos con las pizarras digitales interactivas aplicando programas educativos apropiados.

El correcto funcionamiento del sistema auditivo permite realizar funciones importantes como oír, escuchar, comprender y comunicar. También facilita el desarrollo de operaciones cognitivas como la atención y la memoria. Los estudios realizados en los últimos años inciden en la relación existente entre la calidad de la entrada sensorial y la eficiencia del desarrollo cognitivo y del lenguaje.

Respecto a la discriminación auditiva, son muchos los programas que encontramos en la Red que nos permitan ayudar a los niños desde muy pequeños a que aprendan a discriminar e interpretar estímulos auditivos.

Ya sabemos, como indican Ferre e Irabau (2002), que el cuerpo calloso une los dos hemisferios cerebrales e interviene de forma muy directa en la memoria, junto con las áreas corticales cerebrales.

La técnica de los "Bits de inteligencia" es un ejemplo clásico para trabajar lenguaje y memoria. Y como indica Portellano (2005), una técnica que permite retener la información aprendida. Un bit es la unidad mínima de información visual y auditiva. Un Bit de Inteligencia es un bit de información que se hace mediante una ilustración o dibujo que debe ser preciso, aislado, no ambiguo y nuevo.

La información entra en el cerebro simultáneamente por la vista y el oído. La imagen visual contribuye a la memorización visual y para ello utilizamos el hemisferio derecho. La información auditiva incide en la memoria auditiva para lo que se activa el hemisferio izquierdo. Cuando ambos estímulos están asociados activan la función del cuerpo calloso y la información forma una unidad cognitiva.

Podemos seguir haciendo un repaso de los diferentes programas o aplicaciones digitales que nos ayuden para el desarrollo de la atención, de las habilidades visuales, auditivas, táctiles, de lateralidad, de la memoria, del sentido espacio-temporal, de las habilidades superiores del pensamiento, de las inteligencias múltiples, de la creatividad y de las funciones ejecutivas, etc.

La idea es que entendamos que debemos interpretar que la tecnología nos brinda un apoyo indiscutible para desarrollar las habilidades y reforzar el proceso de mejora de las dificultades.
L O S
A V A N C E S
TE C NOLÓ G I C O S
P A R A
PERSONALIZAR EL APRENDIZA JE

Simplemente dos ejemplos que sirvan para reflejar como los avances tecnológicos suponen una aportación a la Educación.

Emotiv Insight es un dispositivo electrónico inalámbrico que lee las ondas cerebrales y las traduce en datos significativos que todos puedan entender. La diadema supervisa la actividad cerebral y captura los datos de EEG. Emotiv Insight es un EEG portátil con 5 canales que registra las ondas cerebrales y las traduce mediante algoritmos propios 
en datos significativos. Este dispositivo portátil rastrea y monitorea la actividad cerebral y nos muestra cómo el cerebro está cambiando en tiempo real.

Este set neurológico puede medir y ayudar a mejorar la atención (concentración), el interés (compromiso), el entusiasmo, la relajación y reducir los niveles de estrés. Su utilización en Educación nos puede brindar la oportunidad de trabajar, por ejemplo, con alumnos con trastorno de atención e hiperactividad y ayudarles a controlar las situaciones.

El Electroencefalograma (EEG) es un estudio de la función cerebral que recoge la actividad eléctrica del cerebro. Para recoger la señal eléctrica cerebral se utilizan electrodos colocados en el cuero cabelludo, a los que se añade un líquido conductor para mejorar la recepción de la señal.

La fijación de los electrodos debe realizarse de la mejor manera posible para poder medir una buena señal y no cometer errores, por lo cual se hizo una verificación de la impedancia de los electrodos.

Las Gafas estroboscópicas que mejoran los reflejos y el rendimiento visual. E sistema funciona gracias a unas gafas denominadas Nike Vapor Strobe, provistas de una tecnología que oscurece su cristal bloqueando la visión del sujeto durante breves intervalos de tiempo (efecto estroboscópico). La duración de las interrupciones visuales puede definirse en función de las habilidades perceptivas que se quieren mejorar. Según el equipo de científicos que han desarrollado el sistema, bloquear la visión del sujeto durante periodos cortos de tiempo potencia su memoria visual a corto plazo y mejora la velocidad de reacción ante estímulos visuales.

\section{CONCLUSIONES}

El conocimiento del cerebro a la hora de aplicar la tecnología en el ámbito educativo favorece la tarea docente en su responsabilidad de ayudar a los alumnos en su proceso de aprendizaje. Tener presente que Internet no solo ha cambiado la forma en que las personas producen, crean contenidos, se comunican y se divierten. Altera, además, el funcionamiento del cerebro. El convencimiento de ello nos lleva a entender que la tecnología implica un cambio metodológico que responda a la nueva forma de aprender de nuestros alumnos.

\section{Referencias}

Bavelier, D. y Davidson, R. J. (2013). Brain training: Games to do you good. Nature, 494(7438), 425-426.

Dehaene, S. (2007). Les neurones de la lecture. París. Odile Jacob.

Ferré, J. e Aribau, E. (2008). El desarrollo neurofuncional del niño y sus trastornos. Lebón: Barcelona

Gilbert, I. (2005). Motivar para aprender en el aula: las siete claves de la motivación escolar (Vol. 178). Grupo Planeta (GBS).

Jiménez, J. E., Hernández, S., García, E., Díaz, A., Rodríguez, C. y Martín, R. (2015). Test de atención D2: Datos normativos y desarrollo evolutivo de la atención en educación primaria. European Journal of Education and Psychology, 5(1).

Martín-Lobo, P. (2016). Procesos y Programas de neuropsicología Educativa. (Coordinadora). Madrid: Ministerio de Educación, Cultura y Deporte: Centro Nacional de Investigación e Innovación (CNIIE).

Portellano, J. A. (2005). Introducción a la Neuropsicología. Madrid: McGraw-Hill.

Reyes, M. N. (2016). Niveles de conocimiento sobre neurociencia y su aplicación en los procesos educativos. In Crescendo, 6(2), 104-113. 
Stern E., Grabner R., Schumacher R., Neuper C. y Saalbach H. (2005). Educational Research and Neurosciences-Expectations, Evidence, Research Prospects Education Reform. Vol. 13. 